\title{
Dimensiones emergentes para la creación de una escala de percepción del tratamiento con sustitutivos opiáceos
}

\author{
Óscar Martín lozano Rojas*,**; Izaskun Bilbao Acedos*; francisco González Saiz*; \\ Rosario Ballesta Gómez* \\ *Área de Sistemas de Información e Investigación. Fundación Andaluza para la Atención a las Drogodependencias e Incorporación Social. \\ **Departamento de Psicología. Universidad de Huelva. \\ Enviar correspondencia a: \\ Óscar Martín Lozano Rojas. Avda. Hytasa, Ed. Toledo II, Plta. 3 Ofic. 3. 41006. Sevilla. oscar.lozano@juntadeandalucia.es
}

Recibido: Diciembre de 2006. Aceptado: Febrero de 2007.

\section{RESUMEN}

La medida de la percepción y satisfacción del tratamiento con los programas de sustitutivos opiáceos se ha centrado principalmente en evaluar las propiedades del servicio ofrecido en los centros de tratamiento. Más allá del contexto sanitario, estos programas requieren una implicación de la vida personal y social para su seguimiento. El objetivo de este trabajo es ofrecer un marco teórico de referencia para la construcción de una escala que mida de forma integral la percepción del tratamiento con sustitutivos opiáceos.

Se ha utilizado una muestra de 18 pacientes de un estudio piloto con buprenorfina, transferido desde un programa de tratamiento con metadona, con indicación de retirada del tratamiento -de régimen ambulatorio y residencial-, a los que se les realizó una entrevista semiestructurada. El análisis de los datos se ha realizado mediante la Teoría Fundamentada, y la codificación de las dimensiones y del material se ha triangulado a través de tres analistas especializados. Los resultados obtenidos señalan que la percepción del tratamiento con sustitutivos de los pacientes se vertebra sobre cinco dimensiones principales: el valor salud, la adaptación a la vida cotidiana, el estigma, la retirada del tratamiento y la eficacia percibida. Estos resultados son discutidos con los encontrados en la literatura especializada.

Palabras clave: tratamiento opiáceos, satisfacción de los pacientes, metodología cualitativa, percepción tratamiento.

\section{ABSTRACT}

The measurement of perception and satisfaction with opiate substitute treatment programmes has concentrated mainly on evaluating the properties of the service offered at treatment centres. Beyond the health-care context, these programmes need to become part of the patient's personal and social life for them to be followed. The purpose of this work is to offer a theoretical frame of reference for the construction of a scale for integral measurement of patient perception of opiate substitute treatments.

A sample of 18 outpatient and residential patients in a buprenorphine pilot study, transferred from a methadone treatment programme, who showed indications of abandoning treatment, was given a semi-structured interview. The data analysis was done based on the Grounded Theory, and the dimensions were coded and material triangulated by three specialized analysts. The results show that patient perception of treatment with substitutes is aligned in five main dimensions, value to health, adaptation to daily life, stigma, treatment withdrawal and perceived effectiveness. These results are discussed and compared to those found in specialised literature.

Key words: opiate treatment, satisfaction of patients, qualitative methodology, perception of treatment

\section{INTRODUCCIÓN}

$\mathbf{L}$ a medida de la satisfacción de los pacientes en los servicios de salud juega un papel cada vez más importante en la estructura organizativa de los mismos. La definición de este constructo es compleja y existen casi tantas como autores que lo estudian. En general, el objetivo es tener en cuenta la manera en que la atención es percibida por el usuario y sus reacciones como consecuencia de ello'.

En el ámbito de las drogodependencias, diversos estudios apuntan que este constructo juega un importante papel para la retención de los pacientes en los programas de tratamiento: los pacientes que están más satisfechos presentan mayores tasas de retención en los programas ${ }^{2,3}$. A su vez, el tiempo que los 
pacientes drogodependientes se encuentran en programa está relacionado con los resultados terapéuticos obtenidos ${ }^{4-6}$.

Esta relación adquiere una mayor notoriedad en el caso de los programas con sustitutivos opiáceos, tales como los programas de tratamiento con metadona, LAAM, diactilmorfina, buprenorfina, etc. Algunos de los pacientes que hacen uso de estos servicios pueden ser considerados como pacientes crónicos. Generalmente, su salud y hábitos higiénicos se encuentran deteriorados, y muchos viven en situación de exclusión social. Para estos, los programas de tratamiento con sustitutivos hacen de puente entre los pacientes y el uso de servicios sanitarios. Maximizar la satisfacción con estos programas puede ser una buena estrategia para mejorar la adherencia al tratamiento de los mismos, y así poder llevar un mayor control sobre su salud.

La forma de definir operativamente y medir la satisfacción en el ámbito de las drogodependencias es variada. Entre los cuestionarios más utilizados se encuentra la Escala de Verona de Satisfacción con el Tratamiento con Metadona (VSSS-MT)7, 8 . Ésta consta de cuatro dimensiones que incluyen ítems sobre intervenciones básicas, intervenciones específicas, habilidades de los trabajadores sociales, y habilidades de los psicólogos. El Treatment Perceptions Questionnaire (TPQ $)^{9,10}$ es otro de los instrumentos usados en drogodependencias, e incluye dos dimensiones: una denominada 'percepciones del equipo terapéutico' y otra 'percepciones del programa terapéutico'. Otro instrumento utilizado con muestras de consumidores de drogas es el Client Satisfaction Questionnaire (CSQ) $)^{11}$, formado por una dimensión que mide la satisfacción global.

Estos cuestionarios han sido diseñados para valorar la satisfacción con los servicios recibidos. El contenido de los mismos se centra en medir principalmente aspectos organizativos del servicio prestado, el trato ofrecido por los profesionales y la capacitación de estos últimos.

Pero los programas con sustitutivos opiáceos requieren una alta implicación de la vida social y personal de los pacientes, más allá del contexto sanitario. En estos casos, la satisfacción también depende de la percepción del programa de tratamiento farmacológico que siguen los pacientes y la repercusión que tiene en el desarrollo de su vida diaria.

Para estos programas, la medida de la percepción del tratamiento puede ser un adecuado complemento a su satisfacción con los servicios sanitarios ofrecidos. Así, mientras esta última permite planificar estrategias de mejora en el ámbito sanitario, la percepción del tratamiento farmacológico sirve para acomodar el programa a las circunstancias personales de los pacientes. Por lo tanto, ambas medidas son de interés para ofrecer a los pacientes un programa ajustado a sus necesidades.

En el presente estudio se muestra la construcción de las dimensiones que constituyen la perspectiva del usuario hacia un programa de tratamiento con sustitutivos opiáceos. Siguiendo las recomendaciones de otros autores ${ }^{12,13}$, se ha optado por emplear técnicas de investigación cualitativas. Éstas permiten acercarse al punto de vista del usuario sin un instrumento previo que moldee las respuestas. Por ello, las dimensiones que aparecen emergen del propio discurso de los pacientes.

\section{METODOLOGÍA}

\section{Ámbito}

El estudio se desarrolla en el contexto de una investigación más amplia con un diseño cuasi-experimental pre-post sin grupo control, de pacientes transferidos a buprenorfina desde metadona, con indicación de retirada del tratamiento farmacológico. El estudio está coordinado por la Fundación Andaluza para la Atención a las Drogodependencias y se titula "Tratamiento con buprenorfina para pacientes con dependencia de opiáceos: experiencia piloto en la Comunidad Autónoma Andaluza"14. El objetivo de este estudio más amplio es conocer la efectividad, en términos de mejora de la calidad de vida y reducción de daños asociados a la dependencia, del tratamiento con buprenorfina para una muestra de pacientes con dependencia a opiáceos. Los participantes en el estudio previamente se encontraban en programa de metadona, en régimen ambulatorio y residencial, y posteriormente fueron transferidos a buprenorfina.

\section{Sujetos}

Los entrevistados en el estudio cualitativo se seleccionaron de entre los participantes en la investigación pre-post. Para participar en este estudio debían Ilevar al menos un mes en tratamiento con buprenorfina antes de la entrevista, con el fin de que se encontrasen ya estabilizados en este tratamiento.

Para la conformación de la muestra se seleccionaron intencionalmente a los participantes de acuerdo con los siguientes criterios de heterogeneidad:

1. Contexto del tratamiento: ambulatorio o residencial.

2. Consumo de opiáceos en los seis meses anteriores a la inclusión en el proyecto.

3. Incidencias en el proceso de inducción a buprenorfina: síndrome de abstinencia en alguna de 
Tabla 1. Distribución de la muestra según criterios de heterogeneidad

\begin{tabular}{|c|c|c|c|c|c|c|}
\hline & Recurso & Consumo & Incidencia & $\begin{array}{c}\text { Medicación } \\
\text { adicional }\end{array}$ & Género & VIH \\
\hline 1 & CTA & No & Sí & Sí & $M$ & - \\
\hline 2 & CTA & No & No & Sí & $\mathrm{M}$ & - \\
\hline 3 & CTA & No & No & No & $M$ & - \\
\hline 4 & CTA & No & No & No & $\mathrm{H}$ & + \\
\hline 5 & CTA & No & Sí & No & $\mathrm{H}$ & - \\
\hline 6 & CTA & No & No & No & $\mathrm{H}$ & - \\
\hline 7 & CTA & No & No & No & $M$ & - \\
\hline 8 & CTA & No & Sí & No & $\mathrm{H}$ & + \\
\hline 9 & CTA & No & Sí & No & $M$ & - \\
\hline 10 & $\mathrm{CT}$ & Sí & Sí & No & $\mathrm{H}$ & + \\
\hline 11 & CT & Sí & No & Sí & $\mathrm{H}$ & - \\
\hline 12 & $\mathrm{CT}$ & Sí & Sí & No & $\mathrm{H}$ & - \\
\hline 13 & CT & Sí & No & No & $\mathrm{H}$ & - \\
\hline 14 & CT & Sí & No & No & $\mathrm{H}$ & - \\
\hline 15 & $\mathrm{CT}$ & No & No & Sí & $\mathrm{H}$ & - \\
\hline 16 & $\mathrm{CT}$ & Sí & No & No & $\mathrm{H}$ & - \\
\hline 17 & CT & No & No & No & $\mathrm{H}$ & - \\
\hline 18 & CT & No & No & Sí & $\mathrm{H}$ & + \\
\hline
\end{tabular}

las fases de la inducción con buprenorfina desde metadona, reacción médica o psicológica adversa.

4. Necesidad de medicación adicional durante el proceso de inducción con buprenorfina.

5. Género.

6. Estado serológico frente a VIH.

El número total de entrevistados en este estudio cualitativo fue de 18, correspondiendo 9 a pacientes de comunidades terapéuticas y 9 de régimen ambulatorio. En la tabla 1 se ofrece la distribución de los entrevistados de acuerdo con los criterios de segmentación especificados.

\section{Procedimiento}

La recogida de los datos se realizó mediante una entrevista semi-estructurada con cada participante. Éstas tuvieron lugar en los propios centros de tratamiento, y las realizó una socióloga con experiencia profesional en la realización de entrevistas.

El guión de la entrevista recaba información sobre: a) realización de tratamientos previos con sustitutivos opiáceos (relato de historia de tratamientos previos); b) consumo de drogas durante sus tratamientos previos; c) descripción del tratamiento piloto con buprenorfina (información recibida, expectativas, creencias, actitudes, adaptación a la sustancia, etc.); d) percepción del efecto del nuevo tratamiento en su estilo de vida (relaciones familiares, situación laboral, amistades, ocio y tiempo libre, percepción de estigma...); e) valoración de los profesionales implicados en el proyecto; $y, f$ ) valoración general de la experiencia piloto.

\section{Análisis de los datos}

Las entrevistas fueron íntegramente grabadas y transcritas para su posterior análisis.

El análisis cualitativo se ha realizado a través del Método de Comparación Constante, propuesto por la Teoría Fundamentada ${ }^{15-19}$. Éste consiste en la superposición de tareas de comparación del material recogido hasta la integración y delimitación de las conclusiones teóricas. A través del mismo se busca entender qué dimensiones son importantes para los actores en esa percepción, cómo estructuran y cómo dan significado a esas dimensiones, y cómo han llegado a elaborar la imagen que tienen del fenómeno estudiado. Se trata, por tanto, de una interpretación, realizada a partir de los discursos de los sujetos entrevistados, que nos permita conocer cómo esas personas entienden y dan significado al tema de estudio.

La codificación es una labor de análisis fundamental para obtener una interpretación analítica de los datos; consiste en categorizar fragmentos de datos bajo una etiqueta que simultáneamente resuma y dé cuenta del contenido de cada fragmento. La Teoría Fundamentada distingue cuatro fases de codificación: la "codificación abierta", en la que el investigador no parte de categorías preestablecidas, sino que es un proceso que descubre las categorías y dimensiones 
que emergen de los datos; la "codificación focalizada", en la que se procede a una reducción del número de categorías, reagrupando las categorías iniciales y seleccionando la que tengan una mayor carga conceptual; la "codificación axial", que genera relaciones en torno a los ejes centrales de cada categoría y sus subcategorías, propiedades y dimensiones; y la "codificación teórica", que establece relaciones entre las diversas categorías ${ }^{16,17}$. Por lo tanto, en esta teoría, los términos de "categoría" o "dimensión", no son meramente clasificatorios, sino que son analíticos, es decir, conceptuales o teóricos.

El análisis se ha apoyado en el programa informático de análisis de datos textuales NUDIST-NVIVO.

La definición de dimensiones de análisis y la codificación del material se ha triangulado entre tres analistas especializados en análisis cualitativo.

\section{RESULTADOS}

El discurso de los entrevistados se elabora de acuerdo con su experiencia en programas con susti- tutivos opiáceos, la actitud y expectativas que mantienen hacia estos, y la información que tienen sobre los mismos. En éste se aprecia que la percepción del tratamiento se construye tomando como referencia un 'ideal' de tratamiento que les permitiera 'salir de la droga', 'no tener que buscarse la vida' y 'estabilizar su vida con el tratamiento'.

Sobre esta base, del análisis de las entrevistas emergen cinco dimensiones centrales. Se trata de cinco aspectos globales presentes en la descripción y valoración que los pacientes hacen de su tratamiento. Cada una de esas dimensiones se compone de diversas categorías. La estructura generada de dimensiones y categorías refleja el modo en que se ha dado sentido a los discursos espontáneos de los pacientes sobre la percepción del tratamiento recibido. Ésta constituye una primera definición de los contenidos teóricos para la medición de la percepción subjetiva del tratamiento.

A continuación se describe cada una de las categorías emergentes y su composición interna, y en la tabla 2 se representa la estructura de dimensiones y categorías con un extracto del discurso de los entrevistados que las representa.

Tabla 2. Dimensiones, categorías y enunciados correspondientes a la percepción del tratamiento

\begin{tabular}{|c|c|c|c|}
\hline Constructo & Dimensiones & Categorías & Enunciados \\
\hline \multirow{11}{*}{$\begin{array}{l}\text { PERCEPCIÓN } \\
\text { DEL } \\
\text { TRATAMIENTO }\end{array}$} & \multirow{3}{*}{ Valor Salud } & Efectos físicos & $\begin{array}{l}\text { [el tratamiento]... me estreñía, me quitaba la gana de comer, no tenía líbido... en } \\
\text { definitiva, que me caía mal }\end{array}$ \\
\hline & & Efectos psicológicos & $\begin{array}{l}\text { Yo lo que me veo es más activo y más clara la mente, me deja ser más yo mismo } \\
{[\ldots . . \text {, estás pendiente de todo, se te quedan cosas la mente está despejada... }}\end{array}$ \\
\hline & & Secuelas futuras & $\begin{array}{l}\text { [el tratamiento]... poco a poco me he ido yendo, como que no, es muy poco lo } \\
\text { que se resguarda digamos pa las consecuencias que tiene. }\end{array}$ \\
\hline & \multirow{2}{*}{$\begin{array}{l}\text { Adaptación a } \\
\text { la vida } \\
\text { cotidiana }\end{array}$} & $\begin{array}{l}\text { Recogida del } \\
\text { fármaco }\end{array}$ & $\begin{array}{l}\text {...tienes que estar siempre en un sitio, no tienes libertad de movimientos, no } \\
\text { puedes irte de viaje [...] te limita mucho, te limita mucho... }\end{array}$ \\
\hline & & Tomas diarias & $\begin{array}{l}\text {... le iban a dar una pastilla de estas, pero que le valía pa dos días, o sea se la } \\
\text { tomaba hoy y hasta pasado mañana no se tiene que tomar otra [...] Eso es mejor } \\
\text { ¿no? Más cómodo... }\end{array}$ \\
\hline & \multirow{2}{*}{ Estigma } & Exógeno & No más que ir a por la metadona, ya te hace estar discriminado [... ] es drogadicto \\
\hline & & Endógeno & $\begin{array}{l}\text {...yo no quiero coger la metadona porque allí siempre hay una serie de gente } \\
\text { que está con los mismos problemas que yo... }\end{array}$ \\
\hline & \multirow{3}{*}{$\begin{array}{l}\text { Retirada del } \\
\text { tratamiento }\end{array}$} & $\begin{array}{l}\text { Percepción de } \\
\text { dependencia }\end{array}$ & $\begin{array}{l}\text { [el tratamiento]... que al final dependes [... tú te levantas y si no lo tomas ya lo } \\
\text { echas de menos y yo no quiero depender de nada }\end{array}$ \\
\hline & & $\begin{array}{l}\text { Intensidad de los } \\
\text { síntomas }\end{array}$ & $\begin{array}{l}\text { no tenía ganas de nada, escalofríos, apatía total, pero vamos hacía las cosas } \\
\text { que tenía que hacer, y me puse en } 50 \text { cuando llegué no es que estuviera bien } \\
\text { pero al cabo de dos, tres semanas ya se estabilizó el cuerpo ya }\end{array}$ \\
\hline & & $\begin{array}{l}\text { Síndrome de } \\
\text { abstinencia }\end{array}$ & $\begin{array}{l}\text {...no supieron quitarme, que no, que no supieron quitarme, que eso da más sín- } \\
\text { drome de abstinencia... }\end{array}$ \\
\hline & $\begin{array}{l}\text { Eficacia } \\
\text { percibida }\end{array}$ & Logro de objetivos & $\begin{array}{l}\text {...tengo que quitarme, tengo que salir de aquí [tratamiento] y a parte tengo que } \\
\text { recuperar mi autoestima, tengo que recuperar mis hábitos, tengo que recuperar, } \\
\text { por lo menos salir medio bien }\end{array}$ \\
\hline
\end{tabular}




\section{Valor salud}

Esta dimensión se refiere a los efectos atribuidos al fármaco de tratamiento sobre la salud tanto física como psicológica del paciente. Dichos efectos pueden ser terapéuticos o efectos secundarios no deseados que se derivan de la administración del fármaco. Así mismo, esta categoría contiene también la preocupación referida por los pacientes hacia posibles secuelas futuras del tratamiento farmacológico.

Por lo tanto, dentro de la dimensión etiquetada como "Valor Salud", encontramos las siguientes categorías: a) efectos de tipo físico. Los pacientes valoran la capacidad del fármaco para eliminar la necesidad de consumo y para eliminar los síntomas de abstinencia a opiáceos, así como la duración del efecto del fármaco. Por otro lado, los pacientes refieren la aparición de posibles efectos secundarios, mostrando preocupación por los siguientes efectos no deseados: estreñimiento, sudoración, pérdida del deseo sexual, falta de apetito, nauseas, vómitos, cansancio, pérdida o aumento de peso, efecto sobre la calidad del sueño, percepción del dolor; b) efectos de tipo psicológico. Los pacientes refieren percibir diversos efectos psicológicos derivados del fármaco de tratamiento, tales como apatía, efectos sobre sus capacidades cognitivas, como la atención, la capacidad de comprensión, la memoria, efectos sobre sus habilidades sociales y de comunicación, efecto evasor y euforizante; y, c) secuelas hacia el futuro, que consiste en la preocupación que los pacientes refieren hacia los posibles efectos negativos del fármaco sobre diversos órganos (concretamente mencionan el hígado) y por posibles secuelas que pueda dejar el tratamiento sobre su salud en general.

\section{Adaptación a la vida cotidiana}

Se trata de la forma en que el programa de tratamiento en el que se inscribe el paciente afecta a otros aspectos de su vida (laboral, familiar, de ocio...) y al grado de exigencia que requiere su seguimiento. Dentro de esta dimensión se distinguen dos categorías: a) la recogida del fármaco, que contiene aquellos aspectos relacionados con el modo de dispensación del fármaco y su relación con la vida diaria del paciente: accesibilidad al centro dispensador, horarios, periodicidad de la recogida y necesidad de implicación de familiares; y, b) el número de tomas al día aparece también en los discursos de los entrevistados como un posible elemento que interfiere en su vida cotidiana. Esta subcategoría aparece relacionada con la percepción de dependencia hacia el fármaco de tratamiento, que se verá más adelante.

\section{Estigma}

Se trata de un proceso de elaboración de una identidad estigmatizada, es decir, negativa y etiquetada de forma excluyente, que recae sobre las personas vinculadas al tratamiento. A través del análisis de las entrevistas realizadas se distinguen dos procesos de estigmatización, en función de los actores sociales que lo elaboran: a) estigmatización exógena, referida a la percibida por los usuarios del programa como elaborada por otros actores sociales (ciudadanía, otros usuarios del centro de salud dispensador, consumidores de sustancias psicoactivas ilegales que no están en tratamiento...) y que recae sobre los pacientes en programa; y, b) estigmatización endógena, generada desde el propio grupo de usuarios del programa al percibir la adscripción al tratamiento con sustitutivos opiáceos como un último vínculo simbólico con el mundo de la droga. Esta vinculación se articula sobre la percepción de que la necesidad de realizar el tratamiento es una consecuencia derivada de la adicción, identificándose en fases avanzadas del proceso de tratamiento con un residuo de la dependencia e incluso con una dependencia añadida, de difícil superación. Es, por lo tanto, una percepción estigmatizada del tratamiento con sustitutivos opiáceos que se genera en la cultura de los usuarios del programa.

\section{Retirada del tratamiento}

Esta dimensión se refiere a la dificultad percibida por los pacientes para dejar el tratamiento farmacológico. Las categorías que la componen son: a) sensación de dependencia hacia el fármaco. Esta percepción de depender del fármaco de tratamiento se encuentra relacionada, como se comentó anteriormente, con la adaptación del tratamiento a la vida cotidiana y con la estigmatización endógena del tratamiento. La intensidad percibida de esta dependencia actúa como un motivo para desear la retirada del fármaco. Así mismo, se encuentra relacionada con la percepción de dificultad de dicha retirada; b) intensidad anticipada de los síntomas provocados por la abstinencia, al retirar el fármaco de tratamiento; y, c) duración del síndrome de abstinencia. Existen creencias extendidas entre los pacientes entrevistados sobre la duración del síndrome de abstinencia, así como sobre la duración del periodo durante el cuál puede reaparecer este síndrome tras retirarse un tratamiento farmacológico concreto.

\section{Eficacia percibida}

Se trata de la percepción sobre la capacidad del tratamiento para alcanzar los objetivos pretendidos. Esta dimensión se encuentra, por lo tanto, definida en función de los objetivos que el paciente busque en el 
programa (reducción de daños asociados, desintoxicación, incorporación social...).

\section{DISCUSIÓN}

El presente estudio revela la existencia de cinco dimensiones emergentes en la percepción de los pacientes hacia su programa de tratamiento: el valor salud, entendido como las implicaciones que el tratamiento pueda tener sobre la salud física y psíquica del paciente; la adaptación del tratamiento a la vida cotidiana de los usuarios; el estigma del tratamiento; la facilidad o dificultad para la retirada del tratamiento y la eficacia percibida del mismo para alcanzar sus objetivos.

Como se observa, tres de estas dimensiones manifiestan la percepción de los pacientes en relación a las propiedades farmacológicas del opiáceo utilizado en el programa. Estas serían el valor salud, la retirada del tratamiento y la eficacia percibida. La revisión bibliográfica muestra que, aunque con etiquetas diferentes, el contenido de estas dimensiones aparece en estudios anteriores. En la investigación de Hunt y Rosenbaum ${ }^{20}$ los pacientes en programas de tratamiento con sustitutivos manifestaban su preocupación hacia los efectos fisiológicos y psicológicos no deseados de este fármaco. También hablaban de un rechazo hacia la dependencia al fármaco sustitutivo, interpretado como una segunda adicción que impide estar 'limpio'. En el estudio realizado por Koester et al. ${ }^{21}$ se encuentra que una de las motivaciones principales de los pacientes en estos programas de tratamiento era controlar su adicción a la heroína, y reducir o evitar los riesgos inherentes a la adicción. A su vez, mostraban aprehensión hacia el programa de tratamiento basándose en atribuciones de efectos secundarios físicos no deseados y en el miedo al 'enganche' a esta sustancia. Lenne et al. ${ }^{22}$ refieren que la retirada del fármaco es uno de los factores presentes en la percepción de los pacientes hacia los programas de tratamiento con opiáceos.

Estas dimensiones emergentes relacionadas con las propiedades de los fármacos deberían ser tenidas en cuenta para planificar la diversificación de los programas con sustitutivos opiáceos. En la actualidad, se sabe que no todos los pacientes toleran bien los fármacos disponibles y que un porcentaje de pacientes acaban abandonando el tratamiento. Como ya se ha dicho anteriormente, esto último debe ser evitado pues la retención en el tratamiento se relaciona con una mejor evaluación de resultados. Por ello, se hace necesario ampliar las posibilidades de tratamiento, y permitir que los clínicos prescriban cada fármaco tras analizar las ventajas e inconvenientes que tienen para cada paciente.
En relación a las otras dos dimensiones emergentes, adaptación a la vida cotidiana y el estigma del tratamiento, éstas manifiestan las consecuencias del programa de tratamiento en el plano personal y social de cada paciente.

La primera dimensión, y más concretamente la categoría relativa a la recogida del fármaco, se revela también en otros estudios cómo un importante componente de la satisfacción de los pacientes con su tratamiento. En un trabajo realizado sobre satisfacción con el programa de tratamiento con metadona en el País Vasco ${ }^{23}$, la atadura respecto al centro dispensador aparece como una de las principales quejas de los usuarios. En este estudio se observó un nivel de satisfacción con el programa significativamente más alto entre los usuarios adscritos a la dispensación en farmacias frente a los que recogían la medicación en Centros de Tratamiento de Toxicomanías (CTT). Por otra parte, Trujols y Pérez de los Cobos ${ }^{12}$ realizaron un análisis multivariante para determinar qué variables explicaban la mayor proporción de la varianza de la satisfacción global con el tratamiento con metadona. Los resultados mostraron que el número de horas que los centros dedicaban a la dispensación fue la única variable predictora de satisfacción. Esto hace pensar que la accesibilidad a la dispensación del fármaco es un elemento priorizado en la satisfacción global de los pacientes con el programa de tratamiento.

Respecto al estigma, en el estudio de Koester et al. ${ }^{21}$ se encuentra una relación entre el miedo al 'enganche' al fármaco y la necesidad de elaborar una identidad alejada del estigma que rodea estos tratamientos. Así, al igual que en nuestro estudio, se aprecia un rechazo a la dependencia al fármaco por ser percibida como un último vínculo simbólico con el mundo de la droga y la adicción.

En estas dos últimas dimensiones la estructura organizativa de los programas juega un importante papel. La planificación de estos es difícil, en tanto que los programas con sustitutivos opiáceos requieren estrictos controles para un eficaz funcionamiento. Sin embargo, los esfuerzos en diseñar una adecuada planificación, puede minimizar el impacto que el tratamiento tiene en el desarrollo de la vida diaria, contribuyendo así a la mejora de la calidad de vida de estos pacientes.

Por otro lado, la identificación de las dimensiones vistas representa un primer acercamiento teórico a la definición operativa de una escala para medir la percepción del tratamiento con opiáceos ${ }^{24}$. El empleo de la metodología cualitativa junto a la revisión de la literatura especializada, son procedimientos complementarios implicados en esta fase de construcción de escalas ${ }^{25}$. El primero ha facilitado la detección de aquellos contenidos importantes para la población objetivo. El segundo ha permitido contrastar las 
dimensiones encontradas con los resultados obtenidos por otros autores.

Como se ha visto, algunos de los contenidos incluidos en las dimensiones señaladas en este estudio habían sido previamente señalados por otros autores $^{20-23}$. A diferencia de los anteriores, en este trabajo se han reunido estos contenidos y se han etiquetado dándoles una coherencia teórica, siendo éste el motivo del uso de la teoría fundamentada para el análisis de la información obtenida.

La construcción de una escala con estas dimensiones aportará una herramienta útil para conocer la satisfacción de los pacientes con los tratamientos opiáceos fuera del contexto terapéutico. Esta puede actuar como complemento a la medida de la satisfacción proporcionada por los instrumentos actualmente desarrollados, centrados en medir el servicio prestado. Como ya se describió, los contenidos de éstas se centraban principalmente en aspectos organizativos de los servicios asistenciales.

En cuanto a las limitaciones, el hecho de que la entrevista esté realizada en el contexto de un estudio piloto con buprenorfina, puede explicar que el discurso se centre en aspectos relacionados de manera más directa con el fármaco de tratamiento y con su dispensación. Por ello, es posible que otros aspectos, como el apoyo profesional percibido o la cobertura psicoterapéutica en el proceso de tratamiento ${ }^{23,26}$, no emerjan como dimensiones principales del discurso. Esta ausencia puede deberse a que los sujetos entrevistados elaboraron la percepción del tratamiento en referencia al tratamiento farmacológico -la buprenorfina-, por lo que el discurso se centró en los aspectos relacionados con el propio fármaco y su forma de recogida, y no en el modelo de intervención psicosocial, que se mantuvo sin modificaciones entre ambos tratamientos -metadona y buprenorfina-.

Igualmente, consideramos que la orientación del tratamiento en este estudio hacia la desintoxicación, puede haber dado más relevancia a la dimensión "retirada del tratamiento" de la que podría haber alcanzado en otros contextos terapéuticos.

Realizar nuevas entrevistas a pacientes con perfiles diferentes de los vistos en este estudio y en otros contextos terapéuticos, puede servir para complementar las dimensiones encontradas.

\section{Agradecimientos}

Este trabajo ha sido financiado por el Plan Nacional sobre Drogas, a través de su convocatoria pública de ayudas a la investigación. Los autores desean agradecer especialmente la colaboración del equipo médico de las tres comunidades terapéuticas públicas donde se llevó a cabo la investigación (Centro FADA Los Palacios, Centro FADA Tarifa y Centro FADA Cartaya) y del CTA AMTARIS.

\section{REFERENCIAS}

1. Saturno PJ. La definición de la calidad de la atención. En: Marquet, R. (Ed.). Garantía de calidad en atención primaria en salud. Barcelona: Doyma; 1993.

2. Hser Y, Evans E, Huango D, Anglin DM. Relationship between drug treatment services, retention, and outcomes. Psych Serv. 2004; 55: 767-74.

3. Sanders LM, Trinh C, Sherman BR, Banks SM. Assessment of client satisfaction in a peer counseling substance abuse treatment program for pregnant and postpartum women. Eval Program Plann. 1998; 21: 287-96.

4. De Leon G, Hawke J, Jainchill N, Melnick G. Therapeutic communities. Enhancing retention in treatment using "Senior Professor" staff. J Subst Abuse Treat. 2000; 19: 375-82.

5. Simpson DD, Joe GW, Rowan-Szal GA, Greener JM. Drug abuse treatment process components that improve retention. J Subst Abuse Treat. 1997; 14: 565-72.

6. Simpson DD, Joe GW, Rowan-Szal GA. Drug abuse treatment retention and process effects on follow-up outcomes. Drug Alcohol Depend. 1997; 47: 227-35.

7. Pérez de los Cobos J, Valero S, Haro G, Fidel G, Escuder G, Trujols J, et al. Development and psychometric properties of the Verona Service Satisfaction Scale for methadone treated opioid dependent patients (VSSSMT). Drug Alcohol Depend. 2002; 68: 209-14.

8. Pérez de los Cobos J, Fidel G, Escuder G, Haro G, Sánchez N, Pascual C, et al. A satisfaction survey of opioid-dependent clients at methadone treatment centres in Spain. Drug Alcohol Depend. 2004; 73: 307-13.

9. Marsden J, Nizzoli U, Corbelli C, Margaron H, Torres M, Prada De Castro I, et al. New European instruments for treatment outcome research: reliability of the maudsley addiction profile and treatment perceptions questionnaire in Italy, Spain and Portugal. Eur Addict Res. 2000; 6: 115-22.

10. Rodríguez M, Jiménez-Lerma JM, Iraurgi I, Murua F, Bacigalupe L, Chavarri MR, et al. Evaluación de la satisfacción con el tratamiento en un centro ambulatorio de drogodependencias a través del "Treatment Perception Questionnaire" (TPQ). Adicciones 2002; 14: 1-8.

11. De Wilde E, Hendriks VM. The Client Satisfaction Questionnaire: psychometric properties in a dutch addict population. Eur Addict Res. 2005; 11: 157-62.

12. Trujols J, Pérez de los Cobos J. La perspectiva de los usuarios sobre los tratamientos de mantenimiento con metadona: una revisión centrada en la satisfacción con el tratamiento. Adicciones 2005; 17(supl.2): 181-204. 
13. Stahler GJ, Cohen E. Using ethnographic methodology in substance abuse treatment outcome research. J Subst Abuse Treat. 2000; 18: 1-8.

14. González-Saiz F. Actualización en el tratamiento de la dependencia de opiáceos: experiencias de investigación desde el plan andaluz sobre drogas y adicciones. En: Libro de ponencias: Encuentro de profesionales en drogodependencias y adicciones. Investigación y evaluación (2005). Cádiz: Diputación Provincial de Cádiz; 2005; $17-27$

15. Glaser BG, Strauss AL. The Discovery of Grounded Theory: Strategies for Qualitative Research. New York: Aldine; 1967.

16. Strauss A, Corbin J. Grounded theory methodology: an overview. En: Denzin NK, Lincoln YS. (Eds). Handbook of qualitative research. Thousand Oaks: Sage; 1994

17. Strauss A, Corbin J. Basics of qualitative research: techniques and procedures for developing grounded theory. [Sec.ed.] London: Sage; 1998.

18. Strauss A, Corbin J. Basics of qualitative research: grounded theory procedures and techniques. London: Sage; 1990.

19. Charmaz K. Constructing Grounded Theory. Thousand Oaks: Sage; 2006.

20. Hunt G, Rosenbaum M. "Hustling" within the clinic: Consumer perspectives on methadone maintenance treatment. En: Inciardi JA, Harrison LD. (Eds.). Heroin in the age of crack-cocaine. Thousand Oaks: Sage; 1998.

21. Koester S, Anderson K, Hoffer L. Active heroin injectors'perceptions and use of methadone maintenance treatment: Cynical performance or self-prescribed risk reduction?. Subst Use Misuse. 1999; 34: 2135-53.

22. Lenne M, Lintzeris N, Breen C, Harris S, Hawken L, Mettick $R$, et al. Withdrawal from methadone maintenance treatment: prognosis and participant perspectives. Public Health. 2001; 25: 121-5.

23. Markez I, Iraurgi I, Poo M. Programas de Mantenimiento con Metadona en el País Vasco: dispositivos específicos y oficinas de farmacia. Trastornos Adictivos. 2002; 4: 171-80.

24. Osterlind SJ. Constructing test ítems. Boston: Kluwer Academic Press; 1989.

25. Crocker L, Algina J. Introduction to Classical and Modern Test Theory. New York: Holt Reinhard and Winston; 1986.

26. Arnáez C, Marina PA, Secades R, Fernández JR. Calidad asistencial y satisfacción de las mujeres en tratamiento por drogodependencias en Asturias. Adicciones. 2004; 16: 81-90. 